Please do not remove this page

RMIT

UNIVERSITY

\title{
FFT-OFDM for compressed image transmission: performance using structural similarity
}

Neville, Katrina; Hussain, Zahir

https://researchrepository.rmit.edu.au/esploro/outputs/9921863738601341/filesAndLinks?institution=61RMIT_INST\&index=null

Neville, K., \& Hussain, Z. (2008). FFT-OFDM for compressed image transmission: performance using structural similarity. Proceedings of the 2008 International Conference on Advanced Technologies for Communications, 65-68. https://doi.org/10.1109/ATC.2008.4760519

Published Version: https://doi.org/10.1109/ATC.2008.4760519

Repository homepage: https://researchrepository.rmit.edu.au

(c) 2008 IEEE. Personal use of this material is permitted. However, permission to reprint/republish this material for advertising or promotional purposes or for creating new collective works for resale or redistribution to servers or lists, or to reuse any copyrighted component of this work in other works must be obtained from the IEEE.

Downloaded On 2023/04/26 21:15:41 +1000 


\title{
FFT-OFDM for Compressed Image Transmission: Performance Using Structural Similarity
}

\author{
Katrina L. Neville and Zahir M. Hussain, Senior Member, IEEE \\ School of Electrical \& Computer Engineering, RMIT University, Melbourne, Australia \\ katrina.neville@student.rmit.edu.au; zmhussain@ieee.org
}

\begin{abstract}
While root-mean squared error (RMSE) is a good indicator of error in a received image, it does not always take into account the structure of the image and the way images are perceived by the human eye. Using the newly-proposed structural similarity image measure (SSIM), wavelets previously studied by the authors were analysed again. Results showed a close relationship between RMSE and SSIM, and using a combination of both techniques the Daubechies wavelet family gave slightly better quality images than Biorthogonal family. The auto-correlation of the received images was also used to quantify structural loss.
\end{abstract}

\section{INTRODUCTION}

Wireless data transmission is becoming an everyday phenomena with the widespread use of multimedia enabled mobile phones and wireless Internet. The type of data being transmitted via these devices is also increasing and changing and people are expecting better quality and more reliable transmission of larger and larger quantities of information. To be able to transmit these amounts of data, transmission techniques have had to evolve and compression algorithms are needed to decrease the size of the information being transmitted.

A problem with many types of compression algorithms is loss of data, this will result in smaller data sizes but can also result in degradation of the information being received. This can be particularly bad when the information being transmitted is images. Apart from the error produced from compression is the introduction of error produced from the wireless channel during the transmission of the data. Previous work has focussed on analysing this information loss with respect to mean squared error (MSE) and root mean squared error (RMSE) [1] but recent work in [2] and [3] shows that just using this type of error analysis can give a poor indication of the quality of an image as perceived by the human eye and other methods need to be used to support the MSE and RMSE values.

In this paper two types of error detection algorithms will be analysed, the Mean Structural Similarity (MSSIM) algorithm [2] and two-dimensional auto-correlation, both of which can give a reasonable indication of perceived differences between images. This study will apply these algorithms to images that have been compressed using wavelet compression and transmitted via an FFT-OFDM wireless transmission system. The values calculated using these algorithms will then be compared to the RMSE error calculations previously used to determine the transmission error.

\section{SYSTEM DESCRIPTION}

The system used in this study is shown in Fig (1). This system was designed using MATLAB simulation software. The original image transmitted was the $256 \times 256$ pixel 'woman' image in MATLAB.

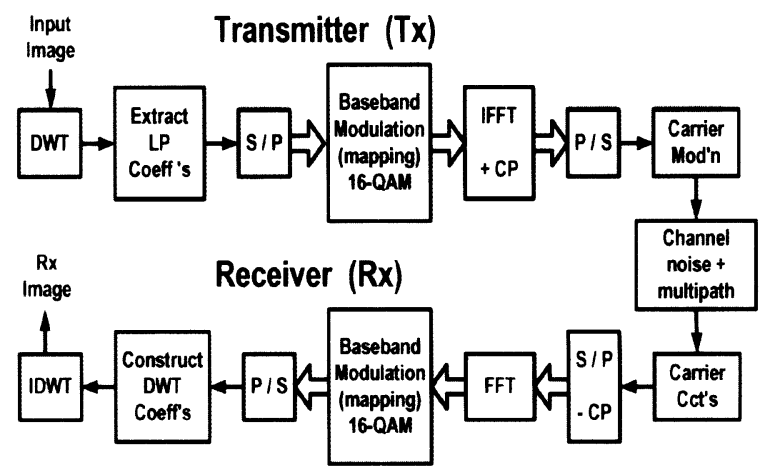

Fig. 1. OFDM transmission system with DWT compression.

\section{A. Transmitter $(T x)$}

The grayscale 'woman' image was input into the system. Using two different types of wavelet families, namely the Daubechies family and the Biorthogonal family, a Discrete Wavelet Transform (DWT) was performed and coefficients extracted.

Two-dimensional wavelet decomposition converts an image into low and high pass subbands by performing a 1dimensional wavelet decomposition on the image twice, first in the horizontal then in the vertical direction [4].

Performing this type of decomposition resulted, visually, in the effect shown in Fig (2) where the image is decomposed into a detail component and two horizontal, vertical and diagonal components with each transform level decreasing the image resolution [4]. The coefficients needed for the experiments performed were the low-pass subbands comprising of the detail and two vertical components of the image as shown in Fig (2). The remaining high-pass coefficients were deleted, this allowed compression to occur. 


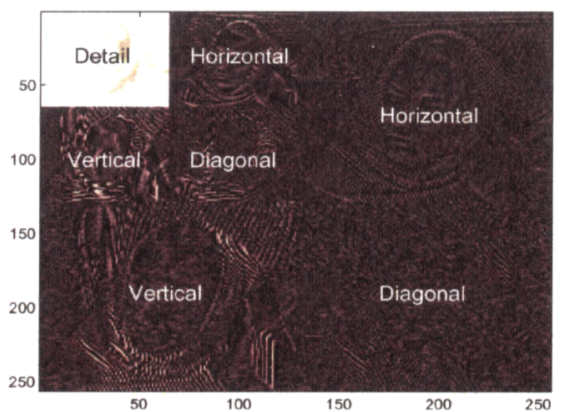

Fig. 2. Two-level wavelet decomposition of 'woman' image.

After the low-pass coefficients were extracted scalar quantisation was applied and the result was converted from decimal to an 8-bit binary string in preparation for modulation.

The modulation scheme chosen for the simulation of the transmission system was FFT-OFDM transmission implemented with 16-QAM. FFT-OFDM is an efficient transmission technique. In the simulated system blocks of $N_{c}$ 16-QAM symbols of the form $D(k)=A(k)+j B(k)$ are taken and an IFFT process is performed on these blocks. The resulting IFFT spectra is then modulated onto orthogonal carriers and transmitted via a fading channel. The discrete-time representation of the signal after IFFT is [5]:

$$
d(n)=\frac{1}{\sqrt{N_{c}}} \sum_{k=-N_{c} / 2}^{k=N_{c} / 2-1} D(k) e^{j 2 \pi \frac{k}{N_{c}} n},
$$

where $N_{c}$ is the number of subchannels, $D(k)$ is the data set being sent and $n \in\left[-N_{c} / 2, N_{c} / 2\right]$.

\section{B. Receiver (Rx)}

At the receiver a channel corrupted and noisy version of the sent data is received. Serial to parallel conversion is applied to this data and an FFT is applied to decode the OFDM blocks, this data is then passed through an equaliser, for the experiments performed for this study perfect channel estimation is assumed at the receiver. Baseband modulation mapping is then performed on this equalised data to determine the most likely 16-QAM symbols received. These symbols are then demodulated, converted back to a serial stream before being converted back to decimal and finally have an inverse wavelet transform performed.

The resulting data was then analysed for structural similarity, RMSE and the correlation between sent and received data.

\section{The Structural Similarity Algorithm}

Mean Squared Error (MSE) is an algorithm commonly used in comparing images and determining the amount of errors between images purely on how many bits have altered from the original image and the one being compared. This algorithm is a good indication of error in a transmission system but experiments have shown that a set of images can have identical MSE values but appear to the human eye to vary substantially in quality [2].

Structural similarity image measure (SSIM) is a measurement system designed to compare images and determine their quality as it would be perceived by the human eye and is based on a comparison of three measures: luminance, contrast and structure in two images.

The luminance measure is defined in [2] as:

$$
l(\mathbf{x}, \mathbf{y})=\left(2 \mu_{x} \mu_{y}+C_{1}\right) /\left(\mu_{x}^{2}+\mu_{y}^{2}+C_{1}\right)
$$

Where $\mu_{x}$ and $\mu_{y}$ are the mean values of the images $\mathbf{x}$ and $\mathbf{y}$ respectively and $C_{1}$ is defined as:

$$
C_{1}=\left(K_{1} L\right)^{2}
$$

where $L$ is the dynamic range of pixel values and $K_{1}<<1$.

Similarly the contrast measure is defined as:

$$
c(\mathbf{x}, \mathbf{y})=\left(2 \sigma_{x} \sigma_{y}+C_{2}\right) /\left(\sigma_{x}^{2}+\sigma_{y}^{2}+C_{2}\right)
$$

where $\sigma_{x}$ and $\sigma_{y}$ are the standard deviations of the images $\mathbf{x}$ and $\mathbf{y}$ respectively and $C_{2}$ is defined as:

$$
C_{2}=\left(K_{2} L\right)^{2} \quad \text { where } K_{2}<<1 .
$$

The structure measure is also defined as:

$$
s(\mathbf{x}, \mathbf{y})=\left(\sigma_{x y}+C_{3}\right) /\left(\sigma_{x} \sigma_{y}+C_{3}\right)
$$

where $C_{3}=C_{2} / 2$ and $\sigma_{x y}$ is defined as

$$
\sigma_{x y}=\frac{1}{N-1} \sum_{i=1}^{N}\left(x_{i}-\mu_{x}\right)\left(y_{i}-\mu_{y}\right)
$$

Finally from [2] the SSIM is defined as:

$$
\operatorname{SSIM}(\mathbf{x}, \mathbf{y})=[l(\mathbf{x}, \mathbf{y})]^{\alpha} \cdot[c(\mathbf{x}, \mathbf{y})]^{\beta} \cdot[s(\mathbf{x}, \mathbf{y})]^{\gamma}
$$

where we considered $\alpha=\beta=\gamma=1$.

\section{2D AUTO-CORRELATION FOR IMAGE COMPARISON}

Cross-correlation is a method used to compare the similarity between two signals. It is a very similar process to convolution except there is no reversal of the functions. The crosscorrelation at time-lag instant $p$ is:

$$
R_{x y}[p]=\sum_{m=-\infty}^{\infty} x(m) y(p+m)
$$

Auto-correlation can be used to detect non-random components in a noisy signal. Random noise that has been autocorrelated results in a delta $(\delta)$ spike at zero lag and virtually zero elsewhere while a deterministic signal that is corrupted with noise should have an auto-correlation function with a peak centred around zero lag and with a symmetrical, finite, non-zero value over a range of time-lag around zero. 


\section{EXPERIMENTAL RESULTS}

MATLAB was utilized to conduct the transmission simulations. Each simulation consisted of 100 realisations so as to average out the crror for each wavelet family and signal to noise ratio simulated.

The channel used in these experiments was a multipath channel and the channel impulse response was assumed to be constant for the entire OFIM frame. The SNR was defined as the ratio of average QAM-symbol energy over noise-energy per QAM-symbol and the power due to the cyclic prefix was ignored since it has no overall effect.

\section{A. RMSE Error}

From previous work [1] RMSE was calculated and analysed for transmission error over four wavelet families: the Iaubechies, Biorthogonal, Symmlets and Coiflets families. From this study it was concluded that the superior families for image compression and transmission were the Daubechies and Biorthogonal families. Therefore for this study those two wavelet families were used as they were not only shown to perform the best as far as RMSE was concerned but also gave better compression ratios which is desirable when transmitting large amounts of data.

Figs (3) and (4) show the RMSE results for image transmission via FFT-OFIM at signal to noise ratios ranging from $5 \mathrm{~dB}$ to $30 \mathrm{~dB}$.

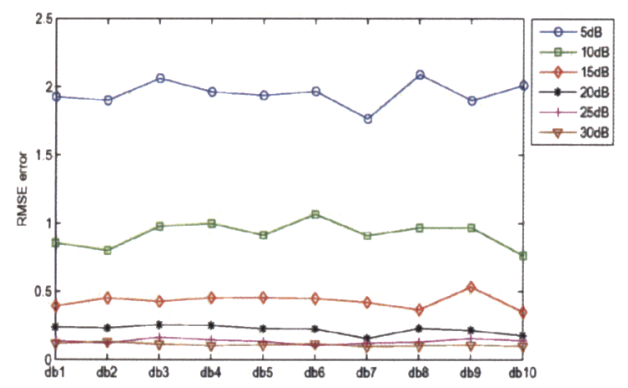

Fig. 3. Wavelet order vs. RMS error (Daubechies family).

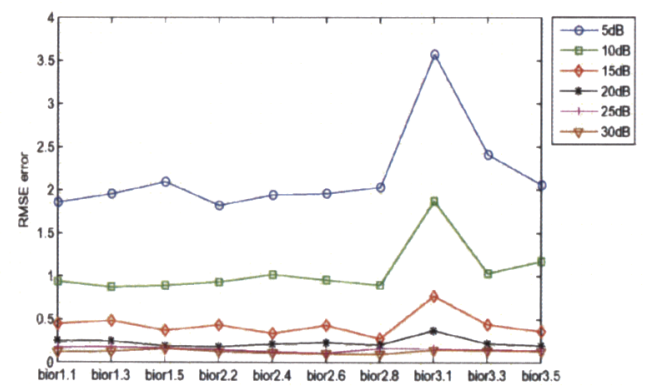

Fig. 4. Wavelet order vs. RMS error (Biorthogonal family).
From these RMSE values it could be seen that both wavelet families had similar performance over all signal to noise ratios with the obvious exception of the Biorthogonal wavelet of order 3.1 which clearly gave a much poorer result for all SNRs.

\section{B. Structural Similarity}

Figs (5) and (6) show the results of the structural similarity simulations from this system. The figures show the percentage similarity between the transmitted and received images. The results for SSIM clearly correlate with the RMSE results with obvious peaks and troughs in RMSE results reflected with peaks and troughs in the SSIM data, particularly noticeable is the severe reduction in structural similarity for the received data for the wavelet Biorthogonal order 3.1.

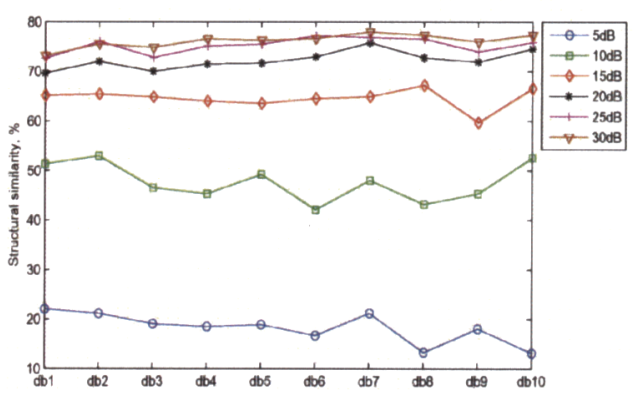

Fig. 5. Wavelet order vs. structural similarity (Daubechies family.

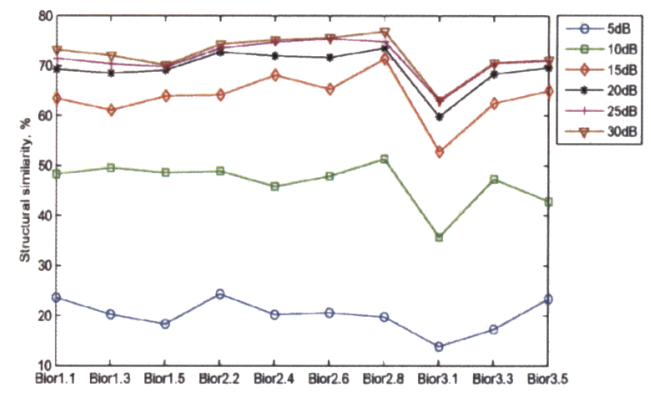

Fig. 6. Wavelet order vs. structural similarity (Biorthogonal family).

The SSIM results show that for a high SNR of $20-30 \mathrm{~dB}$ that, in general, the Daubechies family of wavelets gives better and more consistent structural similarity when comparing images, even so the improvement is only very slight. This result was harder to conclude just from seeing the RMSE values alone.

This same result did not apply to the lower SNR values of 5 $-15 \mathrm{~dB}$ which seemed to produce approximately equal amounts of error for both families of wavelets. From this result it shows there is probably little relationship between transmitted and received images at this SNR due to high corruption from noise. 
This conclusion will be further pursued in Section V$\mathrm{C}$ where auto-correlation of these transmitted and received images will be analysed.

\section{Auto-correlation of transmitted and received images}

Fig (7) shows the auto-correlation of the transmitted image before transmission, it shows the large peak at zero as expected and a symmetrical shape centred around zero indicating the similarity of the images.

Fig (8) was a typical auto-correlation from the received images at $\mathrm{SNR}=5 \mathrm{~dB}$. The tall $\delta$ spike at zero shows there is substantial noise corrupting the data. There is relatively small magnitude symmetry around zero showing minimal deterministic information encapsulated in the data. This result confirms the results in section V-B where the two wavelet families at $\mathrm{SNR}=5 \mathrm{~dB}$ showed great loss of structural similarity.

The normalised correlation error for the system shown in Table I shows the Daubechies wavelet family generally had a smaller error than the Biorthogonal family. Again it is clear that the Biorthogonal 3.1 showed considerable correlation error in relation to the other wavelets.

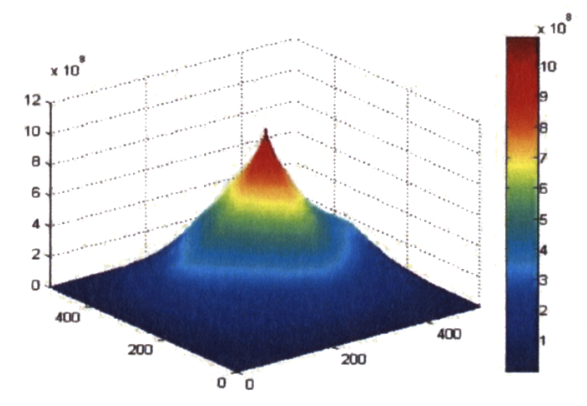

Fig. 7. 2D auto-correlation of transmitted image.

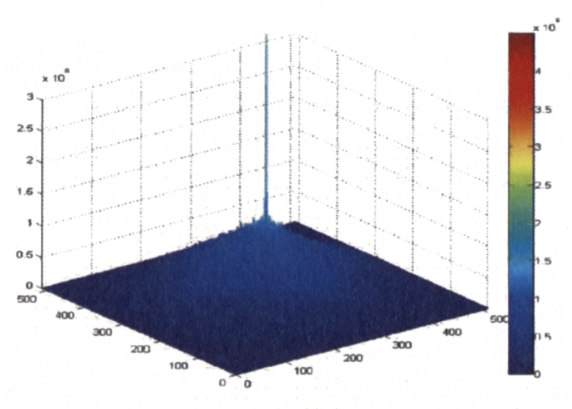

Fig. 8. 2D auto-correlation of received image at $\mathrm{SNR}=5 \mathrm{~dB}$.

\section{CONCLUSION}

The relationship between RMSE and structural similarity is strong. Both methods displayed clearly the wavelets that performed best and worst, with obvious reduction in image quality stemming from using the Biorthogonal 3.1. Using
TABLE 1

NORMALISED CORRELATION ERROR FOR WAVELET FAMILIES.

\begin{tabular}{|c|c|c|c|c|c|c|}
\hline Wavelet & \multicolumn{6}{|c|}{ Correlation error @ SNR of : } \\
& $5 \mathrm{~dB}$ & $10 \mathrm{~dB}$ & $15 \mathrm{~dB}$ & $20 \mathrm{~dB}$ & $25 \mathrm{~dB}$ & $30 \mathrm{~dB}$ \\
\hline \hline Db 1 & 21.9627 & 2.8224 & 1.0072 & 0.0392 & 0.0436 & 1.3906 \\
\hline Db 2 & 12.8184 & 1.3867 & 0.1237 & 1.0675 & 0.0189 & 0.0189 \\
\hline Db 3 & 22.6108 & 2.3987 & 1.7922 & 0.0627 & 0.0595 & 0.0544 \\
\hline Db 4 & 18.2865 & 3.6855 & 0.1327 & 0.5942 & 0.0317 & 0.0317 \\
\hline Db 5 & 9.2416 & 4.4944 & 0.1696 & 0.2956 & 0.0307 & 0.029 \\
\hline Db 6 & 15.6941 & 3.1705 & 2.2698 & 0.4597 & 0.3063 & 0.0162 \\
\hline Db 7 & 23.0748 & 6.3791 & 0.049 & 0.0671 & 0.039 & 0.0089 \\
\hline Db 8 & 40.8365 & 2.4709 & 0.2093 & 0.0211 & 0.0154 & 0.0479 \\
\hline Db 9 & 9.6882 & 5.2221 & 3.93 & 0.1523 & 0.0368 & 0.0368 \\
\hline Db 10 & 26.5865 & 7.9285 & 3.5271 & 0.0667 & 1.4306 & 0.0205 \\
\hline Bior 1.1 & 28.9058 & 2.0934 & 0.2966 & 0.045 & 0.0383 & 0.0383 \\
\hline Bior 1.3 & 16.1595 & 9.64 & 0.185 & 0.0681 & 0.0483 & 0.0551 \\
\hline Bior 1.5 & 12.1973 & 4.1171 & 0.248 & 0.0612 & 0.0759 & 0.0613 \\
\hline Bior 2.2 & 94.8162 & 7.2276 & 0.4798 & 0.8091 & 0.1138 & 0.4866 \\
\hline Bior 2.4 & 11.5994 & 5.1075 & 0.8887 & 0.0748 & 0.0501 & 0.0492 \\
\hline Bior 2.6 & 46.0605 & 4.6653 & 3.7654 & 0.0675 & 0.0568 & 0.0468 \\
\hline Bior 2.8 & 21.6287 & 1.5812 & 0.4707 & 0.0641 & 0.0251 & 0.0196 \\
\hline Bior 3.1 & 139.7415 & 5.5029 & 21.2043 & 1.0415 & 0.2446 & 0.2425 \\
\hline Bior 3.3 & 89.4123 & 10.7735 & 2.8945 & 0.7885 & 0.1488 & 0.1227 \\
\hline Bior 3.5 & 52.5106 & 10.9227 & 3.0877 & 0.1836 & 0.1376 & 0.1376 \\
\hline
\end{tabular}

the SSIM gave a better indication of the wavelet family that produced better quality images at the receiver with high SNRs, showing that the Daubechies wavelets gave a more consistent structural similarity than the Biorthogonal wavelets. Analysis of received images using auto-correlation clearly showed the reduction in performance of both wavelet families at low SNR with very little correlation produced at this SNR.

\section{REFERENCES}

[1] N. Al-Hinai, K. Neville, Z. M. Hussain, and A. Z. Sadik, "Compressed Image Transmission over FFT-OFDM: A Comparative Study," Australiasian Telecommununications Networking and Applications Conference (ATNAC-2007), December 2007.

[2] Z. Wang, A. C. Bovik, H. R. Sheikh, and E. P. Simoncelli, "Image Quality Assessment: From Error Visibility to Structural Similarity," IEEE Transactions on Image Processing, Vol. 13, No. 4, pp. 600 - 612, April 2004.

[3] H. R. Sheikh, M. F. Sabir, and A. C. Bovik, "A Statistical Evaluation of Recent Full Reference Image Quality Assessment Algorithms," IEEE Transactions on Image Processing, Vol. 15, No. 11, pp. 3441 - 3452, November 2006.

[4] D. G. Lee, and S. Dey, "Adaptive and Energy Efficient Wavelet Image Compression For Mobile Multimedia Data Services," IEEE International Conference on Communications, Vol. 4, pp. 2484 - 2490, April/ May 2002.

[5] L. J. Cimini, "Analysis and Simulation of a Digital Mobile Channel using Orthogonal Frequency Division Multiplexing," IEEE Trans. on Communications, Vol. 33, No. 7, pp. 665 - 675, July 1985.

[6] J. A. C. Bingham, "Multicarricr Modulation for Data Transmission: An Idea Whose Time Has Come," IEEE Communications Magazine, Vol. 28, Issue 5, pp. 5 - 14, May 1990. 